\title{
A Forecasting Model Based on High-Order Fluctuation Trends and Information Entropy
}

\author{
Hongjun Guan ${ }^{1}{ }^{(1)}$, Zongli Dai ${ }^{1}$, Shuang Guan ${ }^{2}$ and Aiwu Zhao ${ }^{3, *}$ \\ 1 School of Management Science and Engineering, Shandong University of Finance and Economics, \\ Jinan 250014, China; jjxyghj@126.com (H.G.); studydzl@163.com (Z.D.) \\ 2 Rensselaer Polytechnic Institute, Troy, NY 12180, USA; guans2018@126.com \\ 3 School of Management, Jiangsu University, Zhenjiang 212013, China \\ * Correspondence: aiwuzh@ujs.edu.cn
}

Received: 31 July 2018; Accepted: 3 September 2018; Published: 4 September 2018

\begin{abstract}
Most existing high-order prediction models abstract logical rules that are based on historical discrete states without considering historical inconsistency and fluctuation trends. In fact, these two characteristics are important for describing historical fluctuations. This paper proposes a model based on logical rules abstracted from historical dynamic fluctuation trends and the corresponding inconsistencies. In the logical rule training stage, the dynamic trend states of up and down are mapped to the two dimensions of truth-membership and false-membership of neutrosophic sets, respectively. Meanwhile, information entropy is employed to quantify the inconsistency of a period of history, which is mapped to the indeterminercy-membership of the neutrosophic sets. In the forecasting stage, the similarities among the neutrosophic sets are employed to locate the most similar left side of the logical relationship. Therefore, the two characteristics of the fluctuation trends and inconsistency assist with the future forecasting. The proposed model extends existing high-order fuzzy logical relationships (FLRs) to neutrosophic logical relationships (NLRs). When compared with traditional discrete high-order FLRs, the proposed NLRs have higher generality and handle the problem caused by the lack of rules. The proposed method is then implemented to forecast Taiwan Stock Exchange Capitalization Weighted Stock Index and Heng Seng Index. The experimental conclusions indicate that the model has stable prediction ability for different data sets. Simultaneously, comparing the prediction error with other approaches also proves that the model has outstanding prediction accuracy and universality.
\end{abstract}

Keywords: high-order fluctuation trends; forecasting; information entropy; neutrosophic sets

\section{Introduction}

For stock market forecasts, summarizing the rules that can be used for future predictions from historical data is crucial. At the same time, due to the noise that is contained in the actual data, Song and Chissom [1-3] proposed a fuzzy time series method for general rule extraction. On this basis, some scholars proposed first-order models [4-6], whereas others further studied high-order models [7,8]. These studies not only imply that high-order models can reflect historical trends, but also emphasize the importance of the relationship between historical trends and current state.

However, most of above models summarize the logical relationships between current and historical trends that are only based on discrete states. In fact, in addition to the simple state-to-state relationship, the relationships between the historical states and the current states are also related to other features. For example, Lee et al. [9] suggested that the fluctuation trends in related stock markets are linked with each other. From this point of view, Lee et al. proposed a prediction model that is based on two related stock markets that extended such models to two-factor high-order prediction 
models. In addition to the range of external influences, other internal features of a time series are ignored by traditional autoregressive models. For example, a typical phenomenon, called "callback", occurs after a period of continuous rise, which means that the inconsistencies in historical fluctuation trends might have considerable impacts on future fluctuation trends. In this area, Zhang et al. [10] extracted the inconsistent features of different types of digital signals and selected the optimal feature subsets from 16 entropy features. Jiang et al. [11] proposed a feature fusion model that is based on information entropy (IE) and probabilistic neural network, which uses IE theory to extract the characteristic entropies in vibration signals. These studies inspired us to think that IE can be employed to describe the inconsistency in a period of fluctuation in a time series.

The concept of information entropy was first proposed by Shannon in 1948 [12]. IE is a tool for measuring the degree of ordering of systems. The lower the IE, the less chaos, and the higher the order. Conversely, the larger the IE, the lower the order. IE solves the problem of quantification and describing system complexity and inconsistency. For example, in order to indicate the inconsistency of a battery due to small deviations and uncertainties in its production process, Duan et al. [13] developed a comprehensive evaluation method for battery inconsistency based on IE. Xu et al. [14] proposed the information entropy risk measure, which applied IE to describe the uncertainty in decision risk and the inconsistency of decision information. Based on the uncertainty of rainfall distribution and the inconsistency of information, Wang et al. [15] employed IE to analyze the time-variation in similar-scale rainfall networks in cities. Information entropy has been widely used in many other situations, such as graphics detection [16], algorithm optimization [17], environmental evaluation [18], and big data analysis and mining [19].

In order to fully express the three states of up, down, and inconsistency of a period in history in a logical relationship and to conveniently compare them, the expression variable should have three dimensions. This is similar to the structure of neutrosophic sets (NSs) and the similarity comparisons of different NSs. The NS was introduced by Smarandache [20], which consists of true membership, indeterminacy membership, and false membership. From the perspective of information representation, scholars have proposed three specific concepts that are based on the neutrosophic set: single-valued NSs [21], interval-valued NSs [22], and simplified NSs [23], including single-valued and interval-valued NSs [24]. These concepts seek a more detailed representation of the information, enabling the NSs to express inconsistent information more accurately. At present, NSs have attracted the attention of scholars in various fields. For instance, Abdel-Basse et al. [25] discussed the role of NSs in urban construction and further analyzed its advantages in the construction of imperfect and incomplete information systems. Şahin et al. [26] discussed the application of single-valued NSs in decision making. Van et al. [27] applied NSs to green supplier evaluation and selection. In addition, since similarity is an important method for measuring NSs, similarity was also used in numerous studies [28-30].

As mentioned above, stock market fluctuation is a dynamic nonlinear system. A common question is how complex systems can be synchronized and which parameters have a direct impact on the system's evolution. Researchers think that there are relationships between history, current, and future. It is more likely a small-world network [31]. Therefore, high-order fuzzy time series models have been developed to describe the fluctuations in a time period and generate forecasting rules that are based on discrete high-order states for future prediction. The most problem of these models is the lack of corresponding rules due to limited training samples. This would significantly reduce the accuracy and credibility of such models. The proposed model summarizes the discrete high-order fluctuation states to truth-membership of upper-trend, falsity-membership of upper-trend, and chaos of trends, and then maps them to the three dimensions of truth-membership, falsity-membership, and indeterminacy-membership of a neutrosophic set. It extends existing high-order fuzzy logical relationships to neutrosophic logical relationships. Therefore, the model can employ NS theory to deal with related issues, such as the comparison of high-order states during the location of rules. Compared with traditional discrete high-order fuzzy logical relationships (FLRs), the proposed to 
neutrosophic logical relationships (NLRs) have higher generality and can address the problem that is caused by the lack of rules in the forecasting stage.

Inspired by the above research, we propose a prediction model based on high-order fluctuation trends and information entropy. First, the original time series of the stock market is converted into a fluctuation time series, and then the fluctuation time series is blurred into a fuzzy fluctuation time series according to a predefined label. Second, the IE of its historical fluctuations is calculated based on the probability of different states of each current value. Third, the NS is used to represent the current state and to establish a neutrosophic logical relationships. Fourth, the Jaccard similarity measure is used to seek out similar appropriate logical relationship groups and calculate its expected NSs. Finally, we obtain the desired expected NSs, then the final predicted value is calculated through the process of deneutrosophication. For verification, the proposed model is implemented to forecast Taiwan Stock Exchange Capitalization Weighted Stock Index (TAIEX) and Heng Seng Index (HIS). The experimental conclusions indicate that the model has stable prediction ability for different data sets. Simultaneously, comparing the prediction error with other approaches also proves that the model has outstanding prediction accuracy and universality.

The remainder of this article can be summarized as follows: Section 2 introduces some basic concepts of fuzzy fluctuation time series, IE, deneutrosophication of a neutrosophic set, and neutrosophic logical relationships. Section 3 presents a new prediction method that is based on high-order fluctuation trends and NSs. In Section 4, the TAIEX data set (1997-2005) and HIS (1998-2012) are used to predict future values. Section 5 presents the conclusions of this paper and the potential issues for future research.

\section{Preliminaries}

\subsection{Fuzzy Set (FS)}

Fuzzy set theory was proposed by Zadeh [32], and it has been widely applied in several fields. A brief introduction to the basic concepts of fuzzy set follows.

Definition 1. Denote the universe of discourse as $U=\left\{u_{1}, u_{1}, \ldots, u_{n}\right\}$. A fuzzy set $L=\left\{L_{1}, L_{2}, \ldots, L_{g}\right\}$ in $U$ can be defined by its membership function:

$$
L_{j}=f_{L j}\left(u_{1}\right) / u_{1}+f_{L j}\left(u_{2}\right) / u_{2}+\ldots+f_{L j}\left(u_{n}\right) / u_{n}(j=1,2, \ldots, g)
$$

where $f_{L j:} U \rightarrow[0,1]$ is the membership function of the fuzzy set $L_{j}$, and $f_{L j}\left(u_{i}\right)$ is the membership degree of $u_{i}$ belonging to $L_{j}$, where $f_{L j}\left(u_{i}\right) \in[0,1](I=1,2, \ldots, n, j=1,2, \ldots, g)$. The symbol + is not the conventional operation of addition but union.

Let fuzzy set $L=\left\{L_{1}, L_{2}, \ldots, L_{g}\right\}$ be a finite and fully ordered discrete term set, where $g$ is an odd number in real situations with the first $(g-1) / 2$ elements describe the degree of a property and the last $(g-1) / 2$ elements from the opposite description. For example, when $g=7$, it might represent a set of linguistic variants as: $L=\left\{L_{1}, L_{2}, L_{3}, L_{4}, L_{5}, L_{6}, L_{7}\right\}=\{$ very bad, bad, below fair, fair, above fair, good, very good\}, and so on. The relationship between the element $L_{i}(I=1,2, \ldots, g)$ and its subscript $i$ is strictly monotonically increasing [33], so the function can be defined, as follows: f: $L_{i}=f(i)$. Clearly, the function $f(i)$ is a strictly monotonically increasing function about a subscript $i$.

\subsection{Fuzzy Time Series (FTS)}

Fuzzy time series was proposed by Song and Chissom in 1993 [2]. It has been successfully used to solve many practical problems. The brief introduction of basic concepts of fuzzy time series, as follows.

Definition 2. Let the time series $\{Y(t) \mid t=1,2, \ldots, T\}$, a subset of real number, denote the universe of discourse. According to Definition 1, each element in the time series can be fuzzified to a fuzzy set element $F(t)(t=1,2, \ldots$, 
$T)$, then $\{F(t) \mid t=1,2, \ldots, T\}$ is called a fuzzy time series defined on the time series $\{Y(t) \mid t=1,2, \ldots, T\}$. If there exists a fuzzy relation $R(t-p, t)$, such that

$$
F(t)=F(t-p) \circ R(t-p, t)
$$

where $\circ$ is a max-min composition operator, $F(t)$ is called derived from $F(t-p)$, denoted by the fuzzy logical relationship ( $F L R) F(t-p) \rightarrow F(t) . F(t-p)$ and $F(t)$ are called the left-hand side (LHS) and the right-hand side (RHS) of the FLR, respectively. FLRs with the same LHS can be categorized into an ordered fuzzy logical group (FLG).

If $F(t)$ is caused by $F(t-1), F(t-2), \ldots, F(t-p)$, then the p-order FLR can be represented by: $F(t-p), F(t-p+1), \ldots, F(t-1) \rightarrow F(t)$

\subsection{Fuzzy-Fluctuation Time Series (FFTS)}

Definition 3. Let $\{X(t) \mid t=1,2, \ldots, T\}$ be a time series, where $t$ is the number of the time series. Let $\{Y(t) \mid t=2,3 \ldots T\}$ be a fluctuation time series, where $\{Y(t)=X(t)-X(t-1) \mid t=2,3, \ldots, T\}$. Then the corresponding fuzzy time series $\{Q(t) \mid t=2,3, \ldots, T\}$ is called a fuzzy-fluctuation time series (FFTS).

Definition 4. Let $Q(t)(t=m+1, m+2, \ldots, T, m \geq 1)$ be a FFTS. If $Q(t)$ is caused by $Q(t-1)$, $Q(t-2), \ldots, Q(t-m)$, then the fuzzy-fluctuation logical relationship is represented by:

$$
Q(t-1), Q(t-2), \ldots, Q(t-m) \rightarrow Q(t)
$$

and it is called the m-order fuzzy-fluctuation logical relationship (FFLR) of the fuzzy-fluctuation time series, where $Q(t-1), Q(t-2), \ldots, Q(t-m)$ is called the left-hand side (LHS) and $Q(t)$ is called the right-hand side (RHS) of the FFLR, and $Q(k)(k=t, t-1, t-2, \ldots, t-m) \in L$.

\subsection{Information Entropy}

Information entropy (IE) [12] was firstly defined by Shannon in 1948 as a measure of event uncertainty. Shannon suggested that the smaller the possibility of an incident, the greater the amount of information that it contains. Conversely, the greater the likelihood of an event, the smaller the amount of information. So, the amount of information can be expressed as a function of the probability of occurrence of an event.

\section{Definition 5.}

$$
E=-\sum_{i=1}^{N} p\left(x_{i}\right) \log _{2}\left(p\left(x_{i}\right)\right)
$$

where $p\left(x_{i}\right)$ represents the probability of occurrence of the ith event. In addition, the information entropy must satisfy the following conditions: $\sum_{i=1}^{N} p\left(x_{i}\right)=1,0<p\left(x_{i}\right)<1$ and non-negativity: $E \geq 0$.

\subsection{Neutrosophic Set (NS)}

Neutrosophic set (NS) was proposed by Smarandache [20]. It has been widely used to describe complex phenomena.

Definition 6. Let $X$ be a space of points (objects), with a generic element in $X$ denoted by $x$. A neutrosophic set $A$ in $X$ is characterized by a truth-membership function $T_{A}(x)$, a indeterminacy-membership function $I_{A}(x)$, and a falsity-membership function $F_{A}(x)$. The functions $T_{A}(x), I_{A}(x)$, and $F_{A}(x)$ are real standard or nonstandard subsets of $] 0^{-}, 1^{+}\left[\right.$. There is no restriction on the sum of $T_{A}(x), I_{A}(x)$, and $F_{A}(x)$. 


\subsection{Neutrosophic Logical Relationship (NLR)}

Definition 7. Let $i$ be the subscript of a fuzzy set element $L_{i}(i=1,2, \ldots, g), A(t)$ be the LHS of an m-order FFLR $Q(t-1), Q(t-2), \ldots, Q(t-m) \rightarrow Q(t), P_{A(t)}^{i}$ be the probabilities of corresponding $L_{i}(i=1,2, \ldots$, $g)$ in $A(t) . P_{A(t)}^{i}$ can be generated by:

$$
P_{A(t)}^{i}=\frac{\sum_{j=1}^{m} w_{i, j}}{m} \quad i=1,2, \ldots, g
$$

where $w_{i, j}=1$ if $Q(t-j)=L_{i}$ and 0 otherwise.

Let $A(t)$ be the LHS of a $m$-order FFLR $Q(t-1), Q(t-2), \ldots, Q(t-m) \rightarrow Q(t), E_{A(t)}$ be the entropy of $A(t), P_{A(t)}^{i}$ be the probabilities of corresponding $L_{i}(i=1,2, \ldots, g)$ in $A(t)$. $A(t)$ can be represented by a neutrosphic set $N_{A(t)}$, with the truth-membership function $T_{N_{A(t)}}$, indeterminacy-membership $I_{N_{A(t)}}$, and falsity-membership function $F_{N_{A(t)}}$ defined by:

$$
\left\{\begin{array}{c}
T_{N_{A(t)}}=\sum_{i=1}^{\frac{g-1}{2}} \alpha_{i} P_{A(t)}^{i} \\
I_{N_{A(t)}}=E_{A(t)} \\
F_{N_{A(t)}}=\sum_{i=\frac{g-1}{2}+1}^{g} \beta_{i} P_{A(t)}^{i}
\end{array}\right.
$$

where $\alpha_{i}, \beta_{i}$ are the weights of $L_{i}(i=1,2, \ldots, g)$ in terms of their contribution to represent the corresponding characteristics, $\sum_{i=1}^{(g-1) / 2} \alpha_{i}=1, \sum_{i=\frac{g-1}{2}+1}^{g} \beta_{i}=1$.

The same as the definition of FLG, $N_{A(t)}$ is called the left-hand side (LHS) of a neutrosophic logical relationship (NLR). The similar LHSs of NLRs can be categorized and group their RHSs of corresponding FFLRs into an ordered fuzzy logical group (FLG) $B_{A(t)}$, which can also be represented by a neutrosophic set $N_{A(t)}^{\prime}$, referring to the above method.

$$
\left\{\begin{array}{c}
T_{N_{A(t)}^{\prime}}=\sum_{i=1}^{(g-1) / 2} \alpha_{i} P_{B_{A(t)}}^{i} \\
I_{N_{A(t)}^{\prime}}=E_{B_{A(t)}} \\
F_{N_{A(t)}^{\prime}}=\sum_{i=\frac{g-1}{2}+1}^{g} \beta_{i} P_{B_{A(t)}}^{i}
\end{array}\right.
$$

where $P_{B_{A(t)}}^{i}$ represents the probability of corresponding $L_{i}(i=1,2, \ldots, g)$ in $B_{A(t)}$.

Thus, a neutrosophic logical relationship (NLR) can be defined as: $N_{A(t)} \rightarrow N_{A(t)}^{\prime}$.

Definition 8. Let $N_{A(t 1)}$ and $N_{A(t 2)}$ be two NSs. The Jaccard similarity [34] between $N_{A(t 1)}$ and $N_{A(t 2)}$ in vector space can be expressed as follows:

$$
\begin{aligned}
& J\left(N_{A(t 1)}, N_{A(t 1)}\right) \\
& =\frac{T_{N_{A(t 1)}} T_{N_{A(t 2)}}+I_{N_{A(t 1)}} I_{N_{A(t 2)}}+F_{N_{A(t 1)}} F_{N_{A(t 2)}}}{\left(T_{N_{A(t 1)}}\right)^{2}+\left(I_{N_{A(t 1)}}\right)^{2}+\left(F_{N_{A(t 1)}}\right)^{2}+\left(T_{N_{A(t 2)}}\right)^{2}+\left(I_{N_{A(t 2)}}\right)^{2}+\left(F_{N_{A(t 2)}}\right)^{2}-\left(T_{N_{A(t 1)}} T_{N_{A(t 2)}}+I_{N_{A(t 1)}} I_{N_{A(t 2)}}+F_{N_{A(t 1)}} F_{N_{A(t 2)}}\right)}
\end{aligned}
$$




\subsection{Deneutrosophication of a Neutrosphic Set}

Definition 9. Deneutrosophication of a neutrosophic fluctuation set refers to converting a neutrosophic set $x$ into a fuzzy set $y$ by the following function according to Ali et al. [35]:

$$
f(T(x), I(x), F(x)):([0,1],[0,1],[0,1]) \rightarrow[0,1]
$$

Specifically, in this paper, our equation for deneutrosophication is as follows:

$$
y=\gamma T(x)+\theta I(x)+\delta F(x)
$$

\section{Proposed Model Based on High-Order Fluctuation Trends and Information Entropy}

This paper presents a prediction model that is based on high-order fluctuation trends and information entropy. Different from existing high-order fuzzy time series forecasting models, the proposed model summarizes the discrete high-order fluctuation states into truth-membership of upper-trend, falsity-membership of upper-trend, and chaos of trends. It coincides with the definition of neutrosophic set, which employs the three dimensions of truth-membership, falsity-membership, and indeterminacy-membership to describe a characteristic. Based on the NS expression of high-order fluctuation states, the NS theory can be used to deal with related issues, such as the comparison of high-order states during the location of rules. The most significant contribution of this model is that it extends existing high-order fuzzy logical relationships to neutrosophic logical relationships and it employs information entropy to represent the indeterminacy of fluctuation trends. When compared with traditional discrete high-order FLRs, the proposed NLRs have higher generality and can handle the problem caused by the lack of rules in the forecasting stage. The detailed steps are shown as follow steps and in Figure 1.

Step 1 Construct FFTS for the historical training data.

For each element $X(t)(t=1,2, \ldots, T)$ in the historical training time series, its fluctuation trend is determined by $Y(t)=X(t)-X(t-1)(t=2,3, \ldots, T) . Y(t)$ can be fuzzified into a linguistic set $\left\{L_{1}, L_{2}, L_{3}, L_{4}, L_{5}\right\}=\{$ down, slightly down, equal, slightly up, up $\}$. Let len be the whole mean of all the elements in the fluctuation time series $Y(t)(t=2,3, \ldots, T)$, and we define intervals $u_{1}=[-\infty,-1.5$ len $]$, $u_{2}=[-1.5$ len, -0.5 len $], u_{3}=[-0.5$ len,0.5len $], u_{4}=[0.5$ len, 1.5 len $]$, and $u_{5}=[1.5$ len, $+\infty]$. Then, $Y(t)(t=2,3, \ldots, T)$ can be fuzzified into a fuzzy fluctuation time series $Q(t)(t=2,3, \ldots, T)$.

Step 2 Establish $m$-order FFLRs for the training data set

According to Definition 4, each $Q(t)(t>\mathrm{m})$ in the training set can be represented by its previous $m$ days' fuzzy-fluctuation numbers. Then, the $m$-order FFLRs of the prediction model can be established. Step 3 Calculate fluctuation information entropy

According to the Definition 5, the information entropy of the $m$-order fluctuation can be separately calculated. Among them, $p\left(x_{1}\right), p\left(x_{2}\right), p\left(x_{3}\right), p\left(x_{4}\right)$, and $p\left(x_{5}\right)$, respectively, indicate the probability of occurrence of the linguistic variants $L_{1}, L_{2}, L_{3}, L_{4}$, and $L_{5}$ in the LHS. Then, the information entropy of the $m$-order FFLRs can be obtained according to Equation (4), which will be the element of the indeterminacy membership of the NSs.

Step 4 Convert the LHSs of FFLRs to NSs

Calculate the probability of corresponding $L_{i}(i=1,2, \ldots, 5)$ in the LHSs of training data set, according to Equation (5). Combined with the information entropy of the $m$-order FFLRs obtained in the previous step, convert the left-hands of FFLRs into neutrosophic sets according to Equation (6). 
Step 5 Grouping and optimization

Applying the Jaccard similarity measure method, categorize and group the converted LHSs of FFLRs according their similarities. Convert the RHSs of corresponding FFLRs into neutrosophic sets according to Equation (7). NLRs are obtained from the training data sets for future forecasting.

Step 6 Forecast test time series

The Jaccard similarity measurement method can be used to find the most appropriate NLR for each test data set. According to Definition 9, the RHS of the NLR can be converted to an expectation fuzzy value $Y(i+1)$. Then, calculate the real number of the fluctuation by: $F^{\prime}(i+1)=Y^{\prime}(i+1) \times l e n$. Finally, the predicted value can be obtained from the actual value of the previous day $X(i)$ and the predicted fluctuation value $F^{\prime}(i+1): X^{\prime}(i+1)=X(i)+F^{\prime}(i+1)$.

- Generate fluctuation time series:

$$
\mathrm{Y}(t)=X(t)-X(t-1),(t=2,3, \ldots, T)
$$

- Calculate $_{T}$ the whole mean of the FTS:

$$
\text { len }=\frac{\sum_{t=2}^{T}|\mathrm{Y}(t)|}{T-1}
$$

- Fuzzify the FTS to FFTS:

$$
\mathrm{F}(t)= \begin{cases}L_{1} & Y(t) \in[-\infty,-1.5 \text { len }) \\ L_{2} & Y(t) \in[-1.5 \text { len },-0.5 \text { len }) \\ L_{3} & Y(t) \in[-0.5 \text { len, } 0.5 \text { len }) \\ L_{4} & Y(t) \in[0.5 \text { len }, 1.5 \text { len }) \\ L_{5} & Y(t) \in[1.5 \text { len },+\infty)\end{cases}
$$

Establish the fuzzy-fluctuation logical relationships(FFLRs):

$$
\begin{gathered}
Q(m), Q(m-1), \ldots, Q(1) \rightarrow Q(m+1) \\
Q(m+1), Q(m), \ldots, Q(2) \rightarrow Q(m+2) \\
\vdots \\
Q(t-1), Q(t-2), \ldots, Q(t-m) \rightarrow Q(t)
\end{gathered}
$$

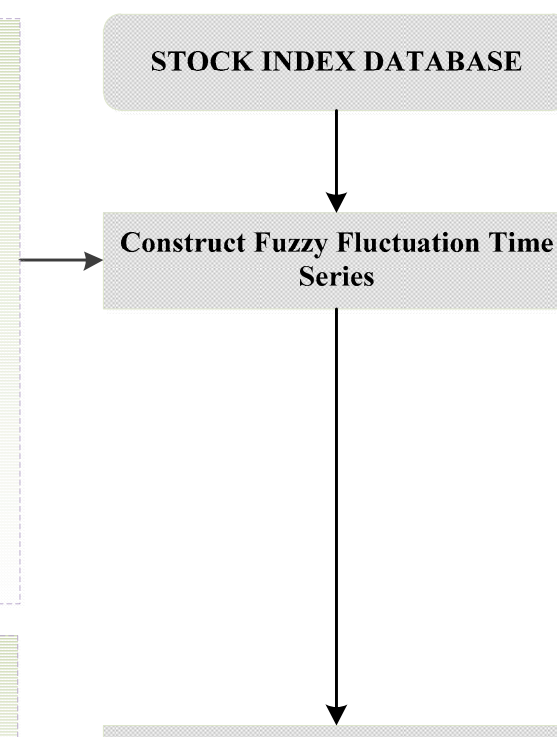

- Calculate the probability of Corresponding Li in the LHSs .

- Convert the LHSs of FFLRs Into neutrosophic sets.

Establish Fuzzy Fluctuation Logic RelationshipS(FFLRs)

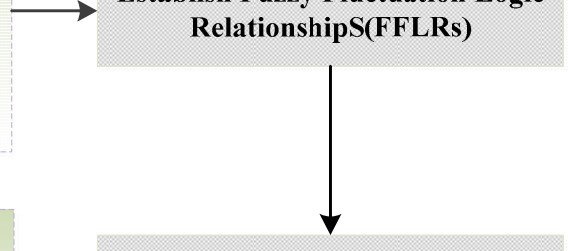

Convert FFLRs To NLRs according to their Jaccard similarity measurement.

- Convert the RHSs of corresponding FFLRs into neutrosophic sets.

- Construct NLRs for future forecasting.
Calculate Similarity And Find The Target Groups

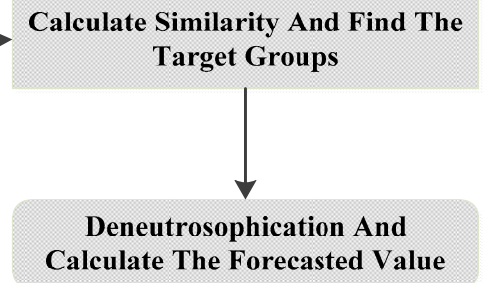

Figure 1. Flow chart of prediction model based on high-order Fuzzy-Fluctuation time series (FFTS) and information entropy (IE). 


\section{Empirical Analysis}

\subsection{Forecasting Taiwan Stock Exchange Capitalization Weighted Stock Index (TAIEX)}

The Taiwan stock market has always been the focus of research in the field of stock market forecasting. The TAIEX is an indicator of the change in the value of Taiwan's overall market stocks, which is seen as a window to the Taiwanese economy. In addition, a large number of studies have used TAIEX data as an example to illustrate their proposed prediction methods [36-41]. To facilitate a comparison with the accuracy of these models, we also used the TAIEX data set to illustrate our proposed method. Specifically, while referring to the general practice of the above studies, the forecasting process in this section is based on TAIEX's 1999 data. Similarly, we also selected data from January 1999 to October 1999 as a training set, and selected from November to December 1999 as a test data set.

Step 1 Construct FFTS for the historical training data

The fluctuation trend is constructed based on the elements of the historical training data set. Then, while using the overall mean of the number of fluctuations in the training data set, the fluctuation trend is blurred to FFTS, e.g., the average value of the training data set for TAIEX. Therefore, len is 85 . Then, $X(1)=6152.43, X(2)=6199.91$, and $Y(2)=X(2)-X(1)=47.48$. Further, $F(2)=L_{4}$, means slightly up. Thus, the training set can be converted to fuzzy fluctuation set.

Step 2 Establish $m$-order FFLRs for the training data set

Different orders directly affect the effect of the prediction. Here, we only need to explain the process, so we take the 9th-order as an example. Then, according to Definition 4, the 9th-order FFLRs of the prediction model were established.

Step 3 Calculating fluctuation information entropy

According to Equation (4), the information entropy of the 9th-order fluctuation time series can be calculated separately. For example, the 9th-order FFLRs on November 1, 1999 can be represented by $\left(L_{3}, L_{3}, L_{2}, L_{4}, L_{3}, L_{3}, L_{3}, L_{3}, L_{5}\right)$, which can be calculated as $p\left(x_{1}\right)=0, p\left(x_{2}\right)=1 / 9, p\left(x_{3}\right)=6 / 9$, $p\left(x_{4}\right)=1 / 9$, and $p\left(x_{5}\right)=1 / 9$. Therefore, its information entropy is 1.4466 , which can be normalized to 0.5306 .

Step 4 Convert the LHSs of FFLRs to NSs

Calculate the probability of corresponding $L_{i}(i=1,2, \ldots, 5)$ in the LHSs of the training data set and then convert the left-hands of FFLRs into neutrosophic sets according to Equation (6).

Step 5 Grouping and optimization

Firstly, apply the Jaccard similarity measure method to categorize and group the converted LHSs of FFLRs. In this example, the threshold similarity value is set to 0.94 . Then, convert the RHSs of the corresponding FFLRs into neutrosophic sets according to Equation (7). The conversion and grouping process is shown in Figure 2. 


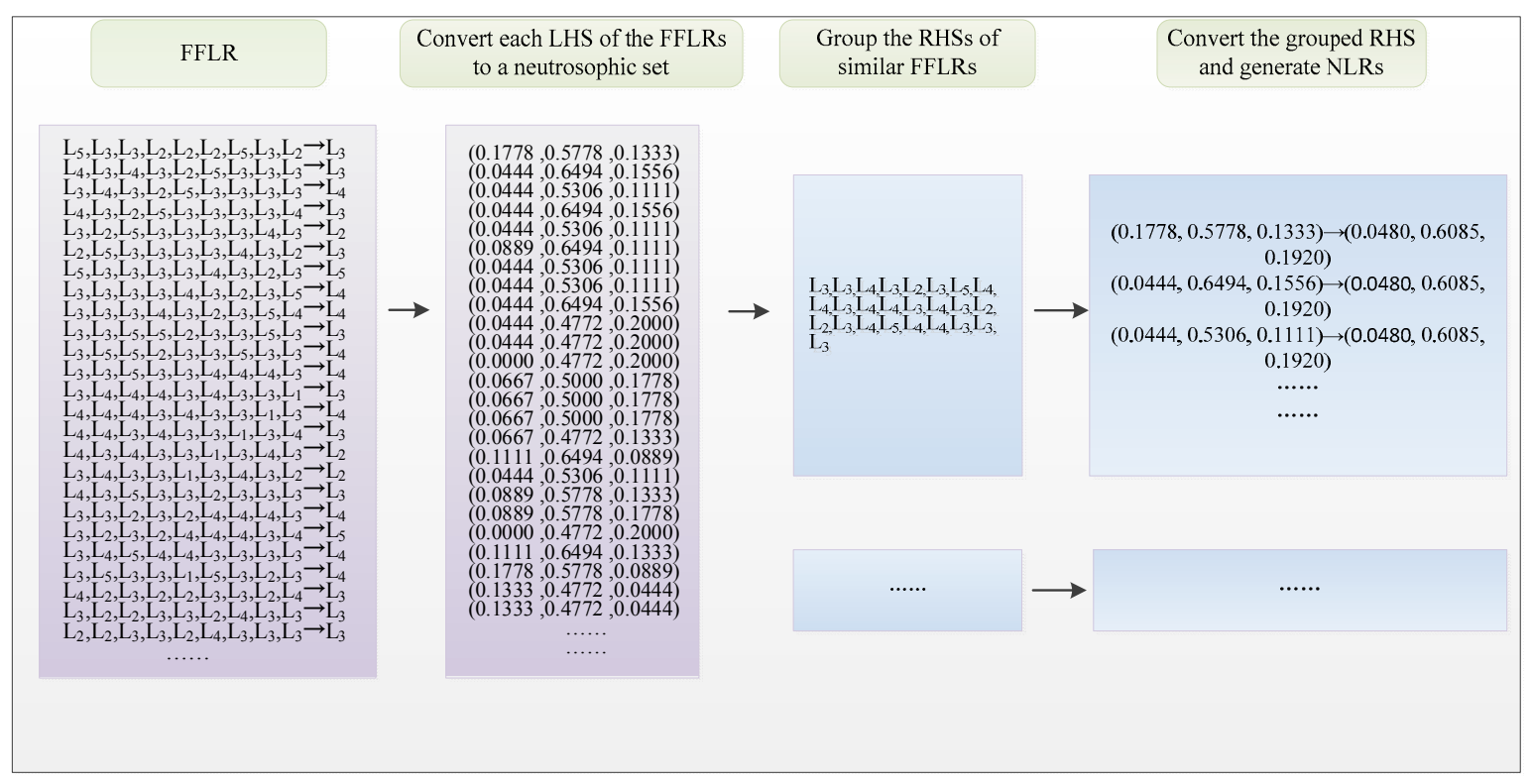

Figure 2. Conversion and group process of fuzzy-fluctuation logical relationship (FFLRs).

Step 6 Forecast test time series

For each test data point, calculate its 9th-order historical fluctuation states and convert them to a neutrosophic set. Locate the NLR with the highest similarity. Then, the corresponding RHS of the NLR is the forecasting neutrosophic value. For example, we chose 1 November 1999 as an example of the test data. The LHS of its fluctuation states is $\left(L_{3}, L_{3}, L_{4}, L_{3}, L_{2}, L_{3}, L_{5}, L_{4}, L_{4}, L_{3}, L_{4}, L_{4}, L_{3}, L_{4}, L_{3}, L_{2}, L_{2}\right.$, $\left.L_{3}, L_{4}, L_{5}, L_{4}, L_{4}, L_{3}, L_{3}, L_{3}\right)$, which can be represented by a neutrosophic set $(0.0480,0.6085,0.1920)$. According to the similarity comparison, the most appropriate NLR for forecasting is $(0.0444,0.5306$, $0.1111) \rightarrow(0.0480,0.6085,0.1920)$.

Then, calculate the predicted fuzzy fluctuation:

$$
Y^{\prime}(i+1)=(-0.0480)+0.1920=0.144
$$

Calculate the real number of the fluctuation:

$$
F^{\prime}(i+1)=Y^{\prime}(i+1) \times \text { len }=0.144 \times 85=12.24
$$

Finally, The predicted value can be obtained from the actual value of the previous day and the predicted fluctuation value:

$$
X^{\prime}(i+1)=X(i)+F^{\prime}(i+1)=7854.85+12.24=7867.09
$$

According to the above steps, we can obtain other prediction results that are shown in Table 1 and Figure 3. 
Table 1. Predicted and actual Taiwan Stock Exchange Capitalization Weighted Stock Index (TAIEX) values from 1 November 1999 to 30 December 1999.

\begin{tabular}{|c|c|c|c|c|c|c|c|}
\hline $\begin{array}{c}\text { Date } \\
(\mathrm{DD} / \mathrm{MM} / \mathrm{YYYY})\end{array}$ & Actual & Forecast & $(\text { Forecast }- \text { Actual })^{2}$ & $\begin{array}{c}\text { Date } \\
(\mathrm{DD} / \mathrm{MM} / \mathrm{YYYY})\end{array}$ & Actual & Forecast & $(\text { Forecast }- \text { Actual })^{2}$ \\
\hline $1 / 11 / 1999$ & 7814.89 & 7867.09 & 2724.84 & $1 / 12 / 1999$ & 7766.20 & 7723.08 & 1859.33 \\
\hline 3/11/1999 & 7580.09 & 7723.14 & $20,462.00$ & $3 / 12 / 1999$ & 7933.17 & 7807.11 & $15,891.12$ \\
\hline $4 / 11 / 1999$ & 7469.23 & 7577.85 & $11,798.99$ & $4 / 12 / 1999$ & 7964.49 & 7932.85 & 1001.33 \\
\hline $5 / 11 / 1999$ & 7488.26 & 7466.21 & 486.03 & $6 / 12 / 1999$ & 7894.46 & 7964.17 & 4859.38 \\
\hline 9/11/1999 & 7362.69 & 7395.82 & 1097.82 & 9/12/1999 & 7738.84 & 7810.16 & 5086.43 \\
\hline $10 / 11 / 1999$ & 7401.81 & 7357.09 & 1999.66 & $10 / 12 / 1999$ & 7733.77 & 7736.72 & 8.67 \\
\hline $11 / 11 / 1999$ & 7532.22 & 7404.90 & $16,210.15$ & $13 / 12 / 1999$ & 7883.61 & 7734.83 & $22,134.74$ \\
\hline $15 / 11 / 1999$ & 7545.03 & 7526.69 & 336.36 & $14 / 12 / 1999$ & 7850.14 & 7882.73 & 1062.35 \\
\hline $16 / 11 / 1999$ & 7606.20 & 7541.78 & 4149.94 & $15 / 12 / 1999$ & 7859.89 & 7849.27 & 112.73 \\
\hline $17 / 11 / 1999$ & 7645.78 & 7606.20 & 1566.58 & $16 / 12 / 1999$ & 7739.76 & 7868.73 & $16,633.26$ \\
\hline $23 / 11 / 1999$ & 8046.19 & 8058.99 & 163.80 & $22 / 12 / 1999$ & 8002.76 & 7938.25 & 4161.84 \\
\hline 24/11/1999 & 7921.85 & 8052.64 & $17,105.57$ & $23 / 12 / 1999$ & 8083.49 & 8002.58 & 6546.57 \\
\hline $25 / 11 / 1999$ & 7904.53 & 7925.99 & 460.58 & $24 / 12 / 1999$ & 8219.45 & 8084.73 & $18,148.43$ \\
\hline $26 / 11 / 1999$ & 7595.44 & 7908.62 & $98,080.84$ & $27 / 12 / 1999$ & 8415.07 & 8224.42 & $36,348.84$ \\
\hline 29/11/1999 & 7823.90 & 7597.74 & $51,147.40$ & 28/12/1999 & 8448.84 & 8418.62 & 913.11 \\
\hline $30 / 11 / 1999$ & 7720.87 & 7823.13 & $10,456.55$ & \multicolumn{3}{|c|}{ Root Mean Square Error(RMSE) } & 102.05 \\
\hline
\end{tabular}

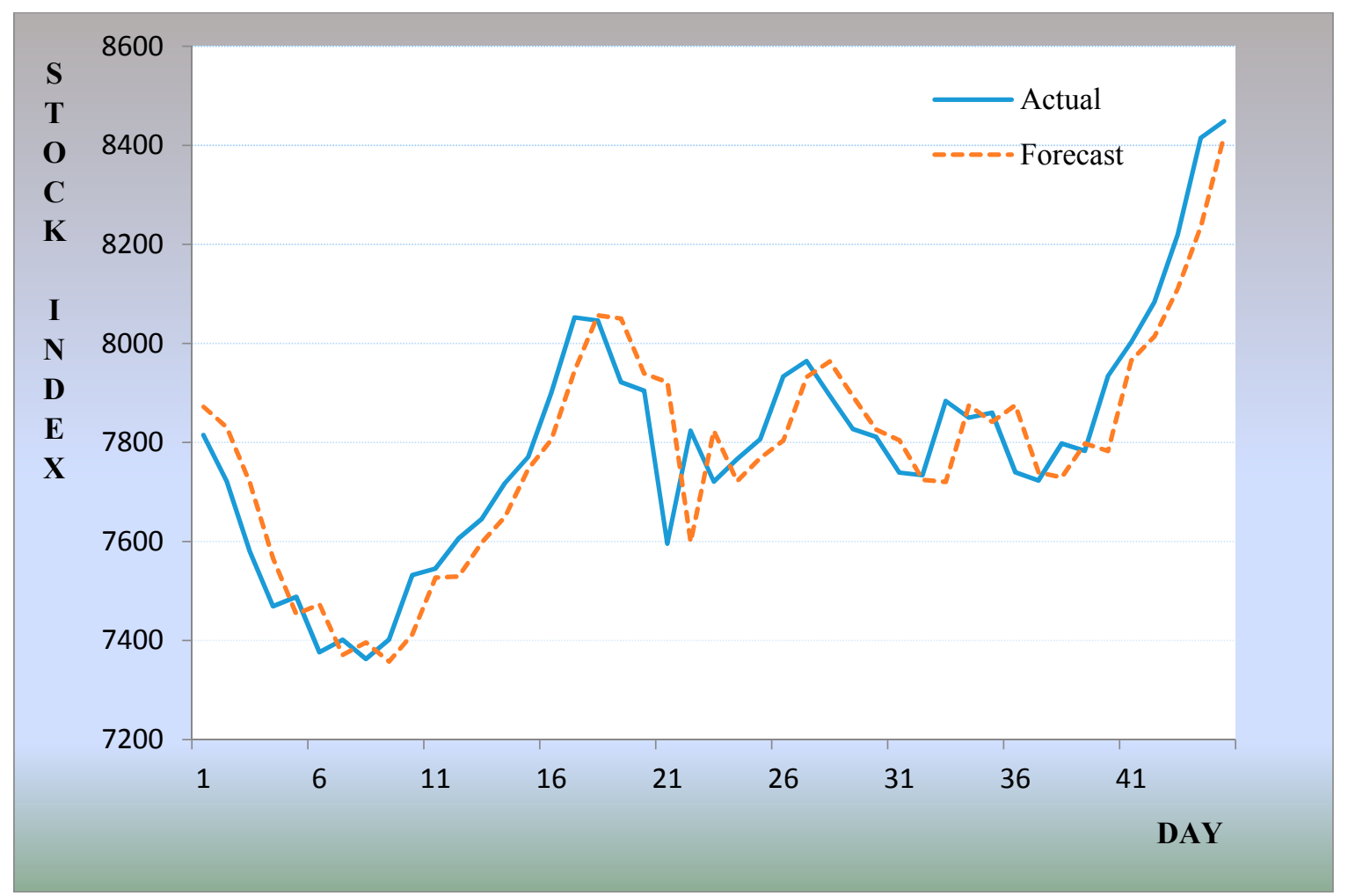

Figure 3. The stock market fluctuation of predicted and actual values for November to December 1999.

In experimental analysis, some of the methods are used to measure the accuracy of prediction in order to quantify the effect of model prediction. These methods are mainly used in the prediction field, including the mean percentage error (MPE), the mean square error (MSE), and the root mean squared 
error (RMSE). Since there is no significant difference in the effect of different error calculation methods, RMSE was chosen as the main formula for error calculation.

$$
R M S E=\sqrt{\frac{\sum_{t=1}^{n}(\text { forecast }(t)-\operatorname{actual}(t))^{2}}{n}}
$$

Since different orders affect the prediction effect, in order to reduce the experimental error and improve the accuracy of the prediction, it was necessary to select the optimal order. Experimental analysis showed that when the order was nine, the predictability of the model is better. Table 2 shows the experimental errors for different years under different orders.

Table 2. Comparison of forecasting errors for different $m$-orders.

\begin{tabular}{cccccccccc}
\hline & \multicolumn{1}{c}{$m$} \\
\cline { 2 - 10 } & $\mathbf{6}$ & $\mathbf{7}$ & $\mathbf{8}$ & $\mathbf{9}$ & $\mathbf{1 0}$ & $\mathbf{1 1}$ & $\mathbf{1 2}$ & $\mathbf{1 3}$ & $\mathbf{1 4}$ \\
\hline RMSE & 102.76 & 102.52 & 102.2 & 102.05 & 102.61 & 102.89 & 102.9 & 102.88 & 103.4 \\
\hline
\end{tabular}

It is important and necessary to accurately predict the trend of fluctuations. Some of the most recent articles have proposed many excellent prediction methods. Therefore, by comparing our method with the previous methods, the advantages and disadvantages of our model can be verified.

Table 3 shows the prediction errors for different methods from 1997 to 2005 . By comparing with the latest research, we found that the overall prediction effect of this method was excellent. For example, the average error of Guan et al.'s model [40] is 94.5, the average error of Cheng et al.'s model [41] is 102.4, and the error of the proposed model is 92.16. Upon further analysis, from the annual prediction error results, we can see that this method can effectively predict the trend from 1997 to 2005 , which is more universal.

Table 3. Performance comparison of prediction root mean squared error (RMSE) with other models for TAIEX from 1997 to 2005.

\begin{tabular}{ccccccccccc}
\hline Method & \multicolumn{10}{c}{ RMSE } \\
\cline { 2 - 9 } & $\mathbf{1 9 9 7}$ & $\mathbf{1 9 9 8}$ & $\mathbf{1 9 9 9}$ & $\mathbf{2 0 0 0}$ & $\mathbf{2 0 0 1}$ & $\mathbf{2 0 0 2}$ & $\mathbf{2 0 0 3}$ & $\mathbf{2 0 0 4}$ & $\mathbf{2 0 0 5}$ & Average \\
\hline Chen and Chang [36] & $\mathrm{N}$ & $\mathrm{N}$ & 123.64 & 131.1 & 115.08 & 73.06 & 66.36 & 60.48 & $\mathrm{~N}$ & 94.95 \\
Chen and Chen [37] & 140.86 & 144.13 & 119.32 & 129.87 & 123.12 & 71.01 & 65.14 & 61.94 & $\mathrm{~N}$ & 106.92 \\
Chen et al. [38] & 138.41 & 113.88 & 102.34 & 131.25 & 113.62 & 65.77 & 52.23 & 56.16 & $\mathrm{~N}$ & 96.71 \\
Cheng et al. [39] & $\mathrm{N}$ & $\mathrm{N}$ & 100.74 & 125.62 & 113.04 & 62.94 & 51.46 & 54.24 & $\mathrm{~N}$ & 84.67 \\
Guan et al. [40] & 141.89 & 119.85 & 99.03 & 128.62 & 125.64 & 66.29 & 53.2 & 56.11 & 55.83 & 94.05 \\
Cheng et al. [41] & $\mathrm{N}$ & 120.8 & 110.7 & 150.6 & 113.2 & 66.0 & 53.1 & 58.6 & 53.5 & 102.4 \\
Our model & 140.33 & 114.35 & 102.05 & 129.97 & 113.32 & 66.26 & 54.66 & 55.19 & 53.33 & 92.16 \\
\hline
\end{tabular}

\subsection{Forecasting Hong Kong Heng Seng Index (HIS)}

HIS is one of the representative indices in Asia. It is not only an important indicator of the Hong Kong stock market price, but is also a stock price index that reflects the most influential trends in the Hong Kong stock market prices. By comparing several authoritative prediction methods, we attempted to verify the universality of the model in other stock markets. Table 4 and Figure 4 compare the different prediction methods from 1998 to 2012. 
Table 4. Performance comparison of prediction RMSE with other models for the Hong Kong Heng Seng Index (HIS) from 1998 to 2012.

\begin{tabular}{|c|c|c|c|c|c|c|c|c|c|c|c|c|c|c|c|c|}
\hline Method & 1998 & 1999 & 2000 & 2001 & 2002 & 2003 & 2004 & 2005 & 2006 & 2007 & 2008 & 2009 & 2010 & 2011 & 2012 & Average \\
\hline Yu [42] & 291.4 & 469.6 & 297.05 & 316.85 & 123.7 & 186.16 & 264.34 & 112.4 & 252.44 & 912.67 & 684.9 & 442.64 & 382.06 & 419.67 & 239.11 & 359.66 \\
\hline Wan et al. [43] & 326.62 & 637.1 & 356.7 & 299.43 & 155.09 & 226.38 & 239.63 & 147.2 & 466.24 & 1847.8 & 2179 & 437.24 & 445.41 & 688.04 & 477.34 & 595.26 \\
\hline Ren et al. [44] & 296.67 & 761.9 & 356.81 & 254.07 & 155.4 & 199.58 & 540.19 & 1127 & 407.89 & 1028.7 & 593.8 & 435.18 & 718.33 & 578.7 & 442.44 & 526.46 \\
\hline Our model & 200.72 & 224.81 & 254.56 & 158.88 & 105.53 & 122.99 & 104.51 & 103.66 & 177.49 & 686.79 & 466.81 & 311.76 & 273.49 & 348.57 & 182.85 & 248.23 \\
\hline
\end{tabular}

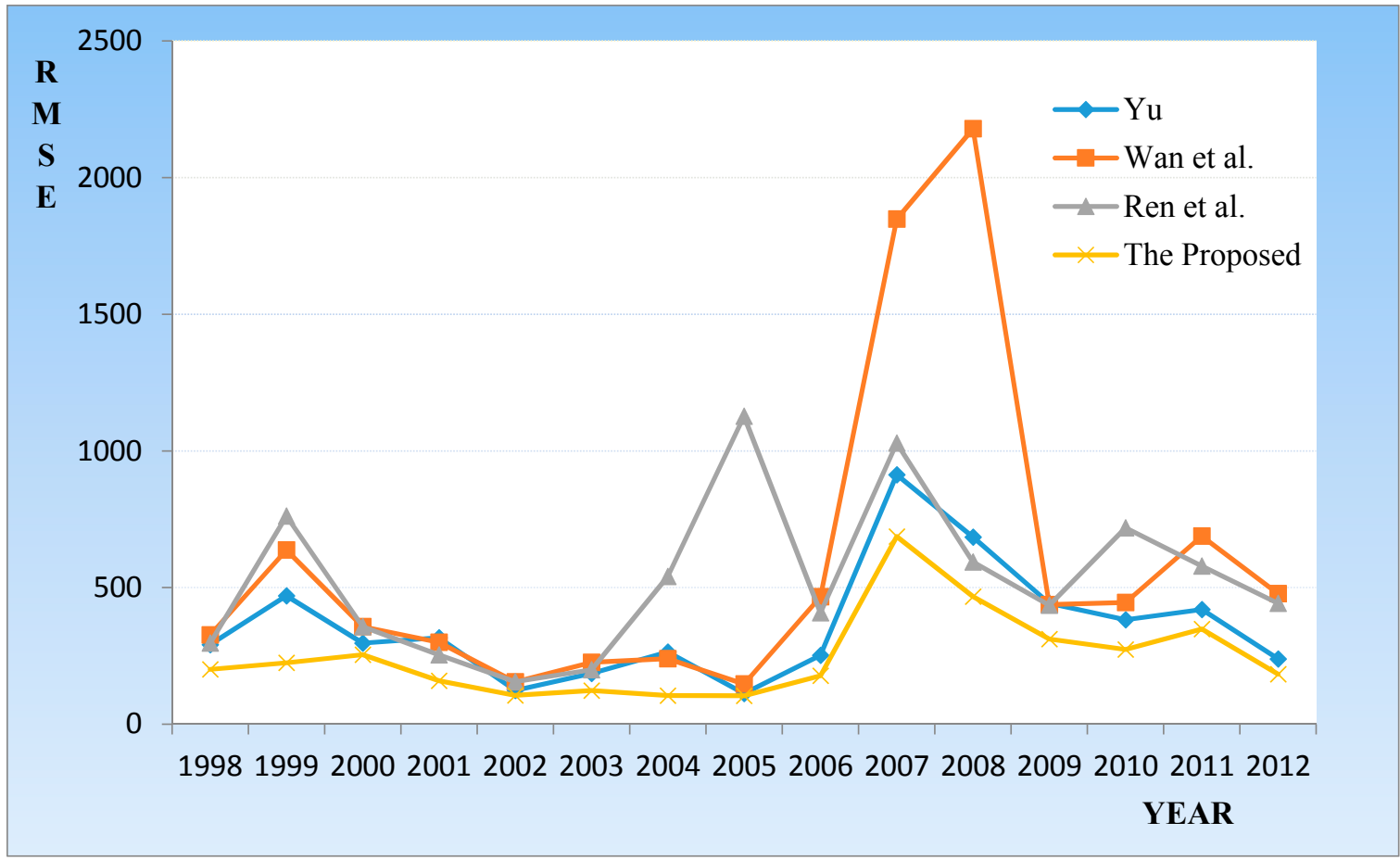

Figure 4. Root mean squared errors (RMSEs) of forecast errors for the Heng Seng Index (HIS) from 1998 to 2012. 
As shown in Table 4, the average prediction error of the Yu [42] method is 359.66, the Wan [43] method is 395.26, and the Ren [44] method is 526.46. The average prediction error of proposed method is 248.23 , which is the smallest error. In addition, it can be seen from Figure 4 that the prediction result of the method is more stable and the prediction effect is more prominent.

\section{Conclusions}

This paper proposed a prediction model that is based on logical rules abstracted from historical dynamic fluctuation trends and the corresponding inconsistencies. During the logical rule training stage, the two dimensions of truth-membership and false-membership of neutrosophic sets were mapped to the dynamic trend states of up and down, respectively. Information entropy was employed to quantify the inconsistency of a period of history and was mapped to the indeterminacy membership of the neutrosophic sets. In the forecasting stage, the similarities among neutrosophic sets were employed to locate the most similar left side of a logical relationship. Therefore, the two characteristics of fluctuation trends and inconsistency assisted with future forecasting. The proposed model was implemented to forecast TAIEX and HIS. The experimental results showed that the model has stable prediction ability for different data sets. Simultaneously, comparing the prediction error with other approaches also proved that our model has outstanding prediction accuracy and universality. This study used two datasets, TAIEX and HIS. Hence, the next study requires data from other stock markets to further validate the model. This study discussed high-order FFTS to characterize the historical fluctuation trends and high-order information fluctuation entropy to measure the inconsistency of historical fluctuations. While considering the relationships among high-order history, current and future, it is likely to use network theory to handle such forecasting problems. In future research, flexible network models should be constructed and more network approaches should be introduced to this area.

Author Contributions: Data curation, Z.D.; Supervision, H.G.; Validation, H.G., S.G. and A.Z.; Writing-original draft, Z.D.; Writing-review \& editing, A.Z.

Funding: This research received no external funding.

Conflicts of Interest: The authors declare no conflicts of interest.

\section{References}

1. Song, Q.; Chissom, B.S. Forecasting enrollments with fuzzy time series-Part I. Fuzzy Sets Syst. 1993, 54, 1-9. [CrossRef]

2. Song, Q.; Chissom, B.S. Fuzzy time series and its models. Fuzzy Sets Syst. 1993, 54, 269-277. [CrossRef]

3. Song, Q.; Chissom, B.S. Forecasting enrollments with fuzzy time series-Part II. Fuzzy Sets Syst. 1994, 62, 1-8. [CrossRef]

4. Pritpal, S.; Gaurav, D. A hybrid fuzzy time series forecasting model based on granular computing and bio-inspired optimization approaches. J. Comput. Sci. 2018, 1877-7503.

5. Che-Ngoc, H.; Tai, V.V.; Huynh-Le, Q.C.; Ho, V.; Nguyen-Trang, T.; Chu-Thi, M. An Improved Fuzzy Time Series Forecasting Model. Available online: https://www.researchgate.net/publication/321947685/ download (accessed on 3 September 2018).

6. Guo, H.; Pedrycz, W.; Liu, X. Fuzzy time series forecasting based on axiomatic fuzzy set theory. Neural Comput. Appl. 2018, 3, 1-12. [CrossRef]

7. Gautam, S.S.; Singh, S.R. A refined method of forecasting based on high-order intuitionistic fuzzy time series data. In Progress in Artificial Intelligence; Springer: Berlin, Germany, 2018; pp. 1-12.

8. Pannu, H.S.; Tripathi, A. High-order fuzzy time series forecasting model for advance prediction of temperature. In Proceedings of the International Conference on Inventive Computation Technologies, Coimbatore, India, 26-27 August 2016; pp. 1-6.

9. Lee, L.W.; Wang, L.H.; Chen, S.M.; Leu, Y.-H. Handling forecasting problems based on two-factors high-order fuzzy time series. IEEE Trans. Fuzzy Syst. 2006, 14, 468-477. [CrossRef] 
10. Zhang, Z.; Li, Y.; Jin, S.; Zhang, Z.; Wang, H.; Qi, L.; Zhou, R. Modulation Signal Recognition Based on Information Entropy and Ensemble Learning. Entropy 2018, 20, 198. [CrossRef]

11. Jiang, Q.; Shen, Y.; Li, H.; Xu, F. New Fault Recognition Method for Rotary Machinery Based on Information Entropy and a Probabilistic Neural Network. Sensors 2018, 18, 337. [CrossRef] [PubMed]

12. Núñez, J.A.; Cincotta, P.M.; Wachlin, F.C. Information entropy. Celest. Mech. Dyn. Astron. 1996, 64, 43-53. [CrossRef]

13. Duan, B.; Li, Z.; Gu, P.; Wang, Y.; Wu, J.; Wang, L.; Zou, X.; Liu, J.; Zou, Y.; He, R. Evaluation of battery inconsistency based on information entropy. J. Energy Storage 2018, 16, 160-166. [CrossRef]

14. Xu, X.H.; Luo, X. Information entropy risk measure applied to large group decision-making method. Soft Comput. 2018, 1-11. [CrossRef]

15. Wang, W.; Wang, D.; Singh, V.P.; Wang, Y.; Wu, J.; Wang, L.; Zou, X.; Liu, J.; Zou, Y.; He, R. Optimization of rainfall networks using information entropy and temporal variability analysis. J. Hydrol. 2018, 559, 136-155. [CrossRef]

16. Huo, W.; Huang, Y.; Pei, J.; Zhang, Q.; Gu, Q.; Yang, J. Ship Detection from Ocean SAR Image Based on Local Contrast Variance Weighted Information Entropy. Sensors 2018, 18, 1196. [CrossRef] [PubMed]

17. Liu, Z.; Shang, P. Generalized information entropy analysis of financial time series. Phys. A Stat. Mech. Appl. 2018, 505, 1170-1185. [CrossRef]

18. Liang, X.; Si, D.; Xu, J. Quantitative Evaluation of the Sustainable Development Capacity of Hydropower in China Based on Information Entropy. Sustainability 2018, 10, 529. [CrossRef]

19. Mahdi, O.A.; Pardede, E.; Cao, J. Combination of information entropy and ensemble classification for detecting concept drift in data stream. In Proceedings of the Australasian Computer Science Week Multiconference, Brisband, Queensland, Australia, 29 January-2 February 2018. [CrossRef]

20. Smarandache, F. A unifying field in logic. In Multiple-Valued Logic; IEEE: Piscataway, NJ, USA, 1999; Volume 8, pp. 489-503.

21. Wang, H.; Smarandache, F.; Zhang, Y.Q.; Sunderraman, R. Single valued neutrosophic sets. Rev. Air Force Acad. 2010, 4, 410-413.

22. Wang, H.; Smarandache, F.; Zhang, Y.Q.; Sunderraman, R. Interval Neutrosophic Sets and Logic: Theory and Applications in Computing; Hexis: Phoenix, AZ, USA, 2005.

23. Ye, J. A multicriteria decision-making method using aggregation operators for simplified neutrosophic sets. Intell. Fuzzy Syst. 2014, 26, 2459-2466.

24. Ye, J.; Cui, W. Exponential Entropy for Simplified Neutrosophic Sets and Its Application in Decision Making. Entropy 2018, 20, 357. [CrossRef]

25. Abdel-Basset, M.; Mai, M. The Role of Single Valued Neutrosophic Sets and Rough Sets in Smart City: Imperfect and Incomplete Information Systems. Measurement 2018, 124, 47-55. [CrossRef]

26. Şahin, R.; Liu, P. Correlation coefficient of single-valued neutrosophic hesitant fuzzy sets and its applications in decision making. Neural Comput. Appl. 2017, 28, 1387-1395. [CrossRef]

27. Van, L.H.; Yu, V.F.; Dat, L.Q.; Dung, C.C.; Chou, S.; Loc, N.V. New Integrated Quality Function Deployment Approach Based on Interval Neutrosophic Set for Green Supplier Evaluation and Selection. Sustainability 2018, 10, 838. [CrossRef]

28. Wu, H.; Yuan, Y.; Wei, L.; Pei, L. On entropy, similarity measure and cross-entropy of single-valued neutrosophic sets and their application in multi-attribute decision making. In Soft Computing; Springer: Berlin, Germany, 2018.

29. Uluçay, V.; Deli, I.; Şahin, M. Similarity measures of bipolar neutrosophic sets and their application to multiple criteria decision making. Neural Comput. Appl. 2018, 29, 739-748. [CrossRef]

30. Ye, J.; Du, S. Some distances, similarity and entropy measures for interval-valued neutrosophic sets and their relationship. Int. J. Mach. Learn. Cybern. 2017. [CrossRef]

31. Bucolo, M.; Fazzino, S.; Rosa, M.L.; Fortuna, L. Small-world networks of fuzzy chaotic oscillators. Chaos Solitons Fractals 2003, 17, 557-565. [CrossRef]

32. Zadeh, L.A. The Concept of a Linguistic Variable and its Application to Approximate Reasoning. Inf. Sci. 1974, 8, 199-249. [CrossRef]

33. Herrera, F.; Herrera-Viedma, E.; Verdegay, J.L. A model of consensus in group decision making under linguistic assessments. Fuzzy Sets Syst. 1996, 79, 73-87. [CrossRef] 
34. Fu, J.; Ye, J. Simplified neutrosophic exponential similarity measures for the initial evaluation/diagnosis of benign prostatic hyperplasia symptoms. Symmetry 2017, 9, 154. [CrossRef]

35. Ali, M.; Minh, N.V.; Le, H.S. A Neutrosophic Recommender System for Medical Diagnosis Based on Algebraic Neutrosophic Measures. Appl. Soft Comput. 2017. [CrossRef]

36. Chen, S.M.; Chang, Y.C. Multi-variable fuzzy forecasting based on fuzzy clustering and fuzzy rule interpolation techniques. Inf. Sci. 2010, 180, 4772-4783. [CrossRef]

37. Chen, S.M.; Chen, C.D. TAIEX Forecasting Based on Fuzzy Time Series and Fuzzy Variation Groups. IEEE Trans. Fuzzy Syst. 2011, 19, 1-12. [CrossRef]

38. Chen, S.M.; Manalu, G.M.; Pan, J.S.; Liu, H.C. Fuzzy Forecasting Based on Two-Factors Second-Order Fuzzy-Trend Logical Relationship Groups and Particle Swarm Optimization Techniques. IEEE Trans. Cybern. 2013, 43, 1102-1117. [CrossRef] [PubMed]

39. Cheng, S.H.; Chen, S.M.; Jian, W.S. Fuzzy time series forecasting based on fuzzy logical relationships and similarity measures. Inf. Sci. 2016, 327, 272-287. [CrossRef]

40. Guan, S.; Zhao, A. A Two-Factor Autoregressive Moving Average Model Based on Fuzzy Fluctuation Logical Relationships. Symmetry 2017, 9, 207. [CrossRef]

41. Cheng, C.; Yang, J. Fuzzy time-series model based on rough set rule induction for forecasting stock price. Neurocomputing 2018, 302, 33-45. [CrossRef]

42. Yu, H.K. A refined fuzzy time-series model for forecasting. Phys. A Stat. Mech. Appl. 2005, 346, 657-681. [CrossRef]

43. Wan, Y.; Si, Y.W. Adaptive neuro fuzzy inference system for chart pattern matching in financial time series. Appl. Soft Comput. 2017, 57, 1-18. [CrossRef]

44. Ren, Y.; Suganthan, P.N.; Srikanth, N. A Novel Empirical Mode Decomposition with Support Vector Regression for Wind Speed Forecasting. IEEE Trans. Neural Netw. Learn. Syst. 2016, 27, 1793-1798. [CrossRef] [PubMed]

(C) 2018 by the authors. Licensee MDPI, Basel, Switzerland. This article is an open access article distributed under the terms and conditions of the Creative Commons Attribution (CC BY) license (http:/ / creativecommons.org/licenses/by/4.0/). 